\title{
Review
}

\section{Guidelines for the conduct of clinical trials for spinal cord injury as developed by the ICCP panel: spontaneous recovery after spinal cord injury and statistical power needed for therapeutic clinical trials}

JW Fawcett*,1, A Curt ${ }^{2}$, JD Steeves ${ }^{2}$, WP Coleman ${ }^{3}$, MH Tuszynski ${ }^{4}$, D Lammertse ${ }^{5}$, PF Bartlett ${ }^{6}$, AR Blight ${ }^{7}$, V Dietz ${ }^{8}$, J Ditunno ${ }^{9}$, BH Dobkin ${ }^{10}$, LA Havton ${ }^{10}$, PH Ellaway ${ }^{11}$, MG Fehlings ${ }^{12}$, A Privat ${ }^{13}$, R Grossman ${ }^{14}$, JD Guest ${ }^{15}$, N Kleitman ${ }^{16}$, M Nakamura $^{17}$, M Gaviria $^{13}$ and D Short ${ }^{18}$

${ }^{1}$ Cambridge University Centre for Brain Repair, Robinson Way, Cambridge, UK; ${ }^{2}$ ICORD, University of British Columbia and Vancouver Coastal Health Research Institute, Vancouver, BC, Canada; ${ }^{3}$ WPCMath, Buffalo, NY, USA; ${ }^{4}$ Center for Neural Repair, University of California, San Diego, La Jolla, CA, USA, ${ }^{5}$ Craig Hospital, Englewood, CO, USA; ${ }^{6}$ Queensland Brain Institute, University of Queensland, St Lucia, Queensland, Australia; ${ }^{7}$ Acorda Therapeutics, Hawthorne, NY, USA; ${ }^{8}$ Spinal Cord Injury Center, Balgrist University Hospital, Zurich, Switzerland; ${ }^{9}$ Jefferson Medical College, Thomas Jefferson University, Philadelphia, PA, USA $;{ }^{10}$ Department of Neurology, University of California Los Angeles, Geffen School of Medicine, Neurologic Rehabilitation and Research Program, Los Angeles, CA, USA; ${ }^{11}$ Department of Movement \& Balance, Division of Neuroscience \& Mental Health, Imperial College London, Charing Cross Campus, London, UK, ${ }^{12}$ University of Toronto, Krembil Neuroscience Center, Head Spine and Spinal Cord Injury Program, Toronto Western Hospital, Toronto, Ontario, Canada; ${ }^{13}$ Institut des Neurosciences - CHU St Eloi, INSERM U-583, Montpellier cedex, France; ${ }^{14}$ Department of Neurosurgery, Baylor College of Medicine, Houston, TX, USA; ${ }^{15}$ Department of Neurological Surgery, Lois Pope LIFE Center, Miami, FL, USA; ${ }^{16}$ National Institute of Neurological Disorders and Stroke, NIH, Bethesda, MD, USA, ${ }^{17}$ Department of Orthopaedic Surgery, Keio University, School of Medicine, Tokyo, Japan; ${ }^{18}$ Midlands Centre for Spinal Injuries, Robert Jones and Agnes Hunt Orthopaedic and District Hospital NHS Trust, Oswestry, Shropshire, UK

The International Campaign for Cures of Spinal Cord Injury Paralysis (ICCP) supported an international panel tasked with reviewing the methodology for clinical trials in spinal cord injury (SCI), and making recommendations on the conduct of future trials. This is the first of four papers. Here, we examine the spontaneous rate of recovery after SCI and resulting consequences for achieving statistically significant results in clinical trials. We have reanalysed data from the Sygen trial to provide some of this information. Almost all people living with SCI show some recovery of motor function below the initial spinal injury level. While the spontaneous recovery of motor function in patients with motor-complete SCI is fairly limited and predictable, recovery in incomplete SCI patients (American spinal injury Association impairment scale (AIS) C and AIS D) is both more substantial and highly variable. With motor complete lesions (AIS A/AIS B) the majority of functional return is within the zone of partial preservation, and may be sufficient to reclassify the injury level to a lower spinal level. The vast majority of recovery occurs in the first 3 months, but a small amount can persist for up to 18 months or longer. Some sensory recovery occurs after SCI, on roughly the same time course as motor recovery. Based on previous data of the magnitude of spontaneous recovery after SCI, as measured by changes in ASIA motor scores, power calculations suggest that the number of subjects required to achieve a significant result from a trial declines considerably as the start of the study is delayed after SCI. Trials of treatments that are most efficacious when given soon after injury will therefore, require larger patient numbers than trials of treatments that are effective at later time points. As AIS B patients show greater spontaneous recovery than AIS A patients, the number of AIS A patients requiring to be enrolled into a trial is lower. This factor will have to be balanced against the possibility that some treatments will be more effective in incomplete patients. Trials involving motor incomplete SCI patients, or trials where an accurate assessment of AIS grade cannot be made before the start of the trial, will require large subject numbers and/or better objective assessment methods.

Spinal Cord (2007) 45, 190-205. doi:10.1038/sj.sc.3102007; published online 19 December 2006 
Keywords: spinal cord injury; clinical trial; clinical examination; clinical assessment; power calculation; ASIA; motor system; sensory system

\section{General background and overall goals}

The annual incidence of spinal cord injury (SCI) varies by region from $<20$ per million people to $>50$ per million people (eg, see http://www.campaignforcure.org/ globalsum.htm). However, as life expectancy after SCI approaches that of the able-bodied population, the worldwide number of survivors has continued to grow to over 2 million people.

The list of experimental interventions, therapies, and devices that have been developed in animal models to improve functional outcomes after SCI is extensive; more importantly several of these will need to undergo clinical trials in the near future. Some early stage SCI clinical trials have recently been started and several more are at a late stage of preclinical maturity. In addition, some experimental therapies have been introduced into clinical practice without a clinical trial being completed.

Thus, there was a need for an international forum where all aspects of clinical trial design could be discussed. The International Campaign for Cures of SCI Paralysis (ICCP) decided to support an international workshop of leading SCI researchers, clinical investigators and companies engaged in the development of SCI treatments to discuss the many issues surrounding the translation of relevant research to a clinical trial. This meeting took place in Vancouver on 20-21 February 2004. ${ }^{1}$ One of the meeting outcomes was a vote by the participants to establish a panel to bring forward more detailed guidelines on how to develop future SCI clinical trials.

The ICCP is an affiliation of 'not for profit' organizations, which aims to facilitate the translation of valid treatments for SCI paralysis. The panel's travel and accommodation expenses have been supported by the following ICCP member organizations: Christopher Reeve Foundation (USA), Institut pour la Recherche sur la Moëlle Epinière (FRA), International Spinal Research Trust (UK), Japan Spinal Cord Foundation, Miami Project to Cure Paralysis (USA), Paralyzed Veterans of America (USA), Rick Hansen Man In Motion Foundation (CAN), SpinalCure Australia, and Spinal Research Fund of Australia, with ICORD (International Collaboration On Repair Discoveries) in Vancouver providing all logistical coordination. The membership of the panel is shown on the list of authors for this and the three accompanying papers, with all panel members volunteering their time and effort.

The ICCP SCI Clinical Guidelines Panel elected to direct the initial set of guidelines towards the design of clinical trials for the rapidly increasing number of experimental cell-based and pharmaceutical drug treatments for protection or repair of the injured spinal cord, whether this is at the acute or chronic stage of SCI. The reasons for this focus are because of the substantial risks and potential benefits of these types of treatments, and because some of these types of treatments have either been offered without completing a clinical trial or will soon enter clinical trials.

Over the past 2 decades, a small number of major SCI clinical trials have been undertaken and completed, including investigations of the neuroprotective benefits of Methylprednisolone, ${ }^{1-5}$ GM-1 (Sygen), ${ }^{6,7}$ and Gacyclidine $(\mathrm{GK}-11) .{ }^{8}$ Each of these trials provided valuable data that has been most useful to the ICCP Clinical Guidelines Panel. Of these therapeutic interventions only methylprednisolone given within $8 \mathrm{~h}$ of injury showed statistically significant efficacy for the treatment of SCI, and none are currently used as standard treatments worldwide. Nevertheless, these trials have highlighted some of the difficulties that will be faced by future trials teams. These include the choice of an appropriate and valid primary clinical end point, selection of trial participants, stratification of subjects, and the coordination and standardization of trial protocols across multiple participating centers.

In short, the SCI field has yet to reach agreement on the conduct of a valid and effective SCI clinical trial. This series of articles contains a discussion and set of recommendations on the many factors that must be considered when designing clinical trials in SCI. We hope these initial guidelines will provide a basis for the design of trials and for future revisions leading to continually improving generations of valid SCI clinical trial protocols.

\section{Introduction}

This paper, the first from the ICCP SCI Clinical Guidelines Panel, is concerned with the assessment of the available data on the natural history of the recovery of neurologic and functional outcomes following SCI. The current literature on rates and degrees of spontaneous recovery is reviewed, presented and compared. In addition, calculations have been made from the data from the Sygen trial to estimate the size of study groups that would need to be recruited into a clinical trial to provide statistically significant results, using one of the measures of neurological outcome that was used for the Sygen study.

\section{Spontaneous recovery and trial design}

The general picture of recovery after spinal injury is well known. A proportion of patients with severe sensorimotor loss will achieve a partial or almost complete neurological recovery, particularly if they have some retained neurological function below the level of injury. As time after injury progresses, more definitive 
assessments and predictions of eventual functional outcome can be made, especially in patients with neurologically complete SCI. An experimental treatment that is delivered within $24 \mathrm{~h}$ of SCI would, therefore, be delivered to a group of subjects where many will show significant spontaneous recovery, unrelated to the treatment received. Therefore, to demonstrate statistically significant results, such a trial would have to recruit a large number of participants. Conversely, a trial of an experimental intervention started much later in the course of post-SCI recovery would require fewer subjects because there is by this time little change from baseline in the absence of treatment. It will, therefore, be important for those designing trials to know the relationship between efficacy and how soon after injury the treatment is initiated.

The main objective of this paper is to gather together previously published data that will allow researchers to understand the degree of spontaneous recovery in different patient groups at different times after SCI and to calculate the size of study groups required for a clinical trial beginning at a particular time following injury. As initially published, much of the previous trial data lack the statistical details needed to make these power calculations, because measures of variance were not included in some of the key publications. We have addressed this by re-analysing some of the data from the control group and the combined control and treatment groups of the largest and most detailed trial of a treatment for spinal injury yet attempted, the Sygen trial. $^{6,7}$

A challenge encountered by the panel is that the data have generally not been presented in a format that relates to the probable recovery patterns that are anticipated for many of the treatments currently being contemplated. The current and upcoming SCI clinical trials focus on neuroprotection, axonal regeneration, promotion of neural plasticity or a combination of these mechanisms. It is probable that the principal clinical benefits of these treatments will be seen in the spinal segments immediately below the site of SCI. Data relating to the recovery patterns in these segments, and the relationship between recovery and distance below the lesion therefore become particularly important.

\section{Methods of assessment}

The assessment methodologies used in previous clinical studies fall into two main categories:

(a) Neurological scoring, including measures of: (1) the completeness of the lesion, measured using the AIS impairment scale or equivalent; (2) the level of lesion, defined by the lowest neurologically functional spinal segment; and (3) the grading of the motor and sensory abilities, using the ASIA motor and sensory scores (or an equivalent). These objective neurological scores assess the remaining connections within the spinal cord, but not the functional abilities of the patient, which also depend on motivation, rehabilitation, fitness and other factors affecting the individual.

(b) Functional assessment: evaluation of the subject's ability to perform Activities of Daily Living (ADL). These tests focus on issues related to the rehabilitation of the patient, and may change independently of neurological outcomes or CNS connectivity; patients with the same neurological scores may therefore, have rather different functional abilities.

The clinical trials completed to date have focussed on forms of assessment that provide information on neurological damage (eg AIS grades or ASIA motor and sensory scores). It is likely that these neurological scores will be among the primary outcome measures indicating whether an experimental intervention has demonstrated acceptable risk (safety) and therapeutic activity or beneficial effect in phase 1 and 2 trials. These outcome measures will probably be backed up by further physiological tests of connectivity, as described in the accompanying paper (SCI Trial Guidelines 2). ${ }^{9}$ However, no SCI therapy will be considered effective for the treatment of patients unless it improves the ability of patients to function in their daily routines or activities. Tools and assessment procedures that accurately characterize such benefits need to be incorporated into definitive phase 3 clinical trials.

\section{Sources of data}

The panel reviewed numerous studies and data reports about SCI recovery. Three large, double-blind, placebocontrolled pharmaceutical trials focussed on acute neuroprotection and rehabilitation in SCI are reported in the literature; the National Acute Spinal Cord Injury Study (NASCIS), Sygen and GK-11 trials. ${ }^{2-8}$ The data from the placebo-control groups in these studies allow the natural history of recovery from injury to be examined within the context of a randomized controlled trial (RCT). In addition, there have been a number of studies of long-term outcomes after SCI that provide valuable information on expected recovery rates, ${ }^{10-12}$ as well as some recently unpublished data from the European Multicenter study in Spinal Cord Injury (EMSCI).

1. The series of three NASCIS trials examined treatment with methylprednisolone, naloxone and tirilazad mesalate, all initiated within a few hours of spinal injury. For ethical reasons, only the second trial (NASCIS II) included a true placebo group. The first trial assumed efficacy based on long established anecdotal use, and compared low and relatively high doses of methylprednisolone over a 10-day regimen. When no difference in functional outcome was seen, the ethical hurdle was reduced and the second trial compared three groups: placebo, $23 \mathrm{~h}$ methylprednisolone treatment, and naloxone. Patients were examined on admission to hospital emergency room, mostly 
within $12 \mathrm{~h}$ after injury, and an average time from injury to treatment of $8.9 \mathrm{~h}$. In this second study, ${ }^{2,3}$ 487 patients were enrolled, of whom 171 were in the placebo group, and outcomes measured at 6 weeks, 6 months and 1 year. The third NASCIS study ${ }^{4,5}$ did not have a placebo-control group, because following its apparent efficacy in the NASCIS II study it was considered that to deny some form of methylprednisolone treatment would be unethical.

2. Sygen (GM-1 ganglioside) was originally studied in a single-center pilot trial that recruited 37 patients. A significant drug effect was found ( $\mathrm{P}$ 0.034) in the proportion of patients able to improve by two grades on the AIS (one of 14 on placebo, seven of 14 on Sygen). This trial was followed by a larger study that recruited 760 patients in 28 centers in the US and Canada (see Supplementary Table 1). Patients were started on the study medication within 3 days of injury, usually on day 2 , and outcomes were measured at intervals up to 1 year. All patients received the same regimen of methylprednisolone as in the active group of NASCIS 2, starting within $8 \mathrm{~h}$. The primary outcome measure was the ability to improve at least two grades between the AIS at baseline and the Benzel Scale (an expansion of AIS) at 26 weeks.

3. A prospective phase $2 \mathrm{RCT}$ of an NMDA receptor blocker, Gacyclidine (GK11), as an early neuroprotective treatment (within $2 \mathrm{~h}$ of $\mathrm{SCI}$ ) was completed in France and involved over 250 patients with 67 placebo-control subjects. ${ }^{8}$

4. The USA Model Systems studies ${ }^{11}$ examined outcomes in 3585 patients admitted to several spinal injury centres. The initial examination was performed within 1 week of injury. Of these, motor score changes were measured over 1 year in 1636 patients.

5. Waters et $a l^{10}$ followed the outcome of 61 complete tetraplegic patients with lesions between $\mathrm{C} 4$ and $\mathrm{C} 7$. The patients were first examined upon admission to the spinal injury unit, which was $<30$ days from the time of injury, and followed-up for up to 2 years.

6. Kirshblum et al ${ }^{12}$ undertook a longitudinal assessment of neurological recovery in 987 people living with SCI at 1 and 5 years after SCI.

7. EMSCI is a group of 14 spinal injury centres. The group is developing a set of trial protocols that will be used in the forthcoming trials, including a Novartissponsored trial of an anti-Nogo-A antibody (AT355). The group has detailed spontaneous recovery information on 217 acute traumatic SCI patients that have been clinically followed with repeated neurological assessments extending for 1 year after injury. This data has not been published previously and we provide only a brief summary of their findings. Further information is available on www.emsci.org.

\section{Neurological recovery after SCI}

As outlined above, the ASIA protocols (or equivalents) are the most frequently used assessment tool in SCI clinical trials and survey studies to measure neurological recovery. Two types of measure are included. The completeness of the SCI is graded by the AIS (AIS grades $\mathrm{A}-\mathrm{E}$ ). The motor and sensory abilities of patients are described by the ASIA motor and sensory scores that constitute the International Standards for Classification of SCI. Recovery can be measured by conversions in the AIS and/or by changes in the ASIA motor and sensory scores. By repeated measures, over the continuum from the acute to chronic stage of SCI, the time profile of changes, as well as the absolute amount of neurologic recovery, can be determined.

\section{Completeness of the lesion, measured using the AIS}

The AIS grades patients from A (sensorimotor complete) to B (motor complete, sensory incomplete), C and $\mathrm{D}$ (motor and sensory incomplete), E (normal). The Sygen study used the Modified Benzel classification, which has grades from 1 to 7 . The main difference from the AIS is the expansion of AIS D into three separate Benzel grades. Translation from AIS grades to Benzel is as follows: grade $1=\mathrm{AIS} A, 2=\mathrm{B}, 3=\mathrm{C}, 4=\mathrm{D}, 5=\mathrm{D}$, $6=\mathrm{D}, 7=\mathrm{E}$ (details in Geisler et $^{a} l^{6,7}$ ).

All studies report a considerable degree of conversion in AIS grade over the first year after SCI, from a sensorimotor complete injury (AIS A) to sensory incomplete (AIS B) or a sensory and motor incomplete (AIS C-D) status. Although the data collection in the studies was performed at different times and under rather different conditions and/or trial designs, the findings seem to be robustly similar (Figure 1). The conversion rates vary greatly depending on the grading of the patient at admission to the study. Thus, $80 \%$ of the initial AIS A patients remain as AIS A, with about $10 \%$ converting to AIS B (ie some sensory function) and about $10 \%$ of the initial AIS A patients regaining some motor function (ie AIS C). The data shown in Figure 1 includes both tetraplegic and paraplegic levels of SCI. The EMSCI data on spontaneous AIS conversion percentages indicates some differences between AIS A tetraplegic and paraplegic patients with the tetraplegic patients demonstrating almost twice the percentage of recovery for conversion to AIS B and AIS D from AIS A (Figure 2).

In patients initially assessed as AIS B or AIS C (ie incomplete SCI) the extent of spontaneous recovery was significantly greater compared to AIS A. The spontaneous 1 year neurologic recovery rate varied from study to study, but AIS B conversion to AIS C was between 15 and $40 \%$ and AIS B conversion to AIS D was also reported to be as much as $40 \%$ in two of the three studies. AIS C conversion to AIS D was between 60 and $80 \%$ of all the patients examined. In the Sygen trial, where the AIS D grade was subdivided into three Benzel classifications, $95 \%$ of patients were reported to have shown an improvement of at least one classification level, although few AIS D patients converted to complete normality. 

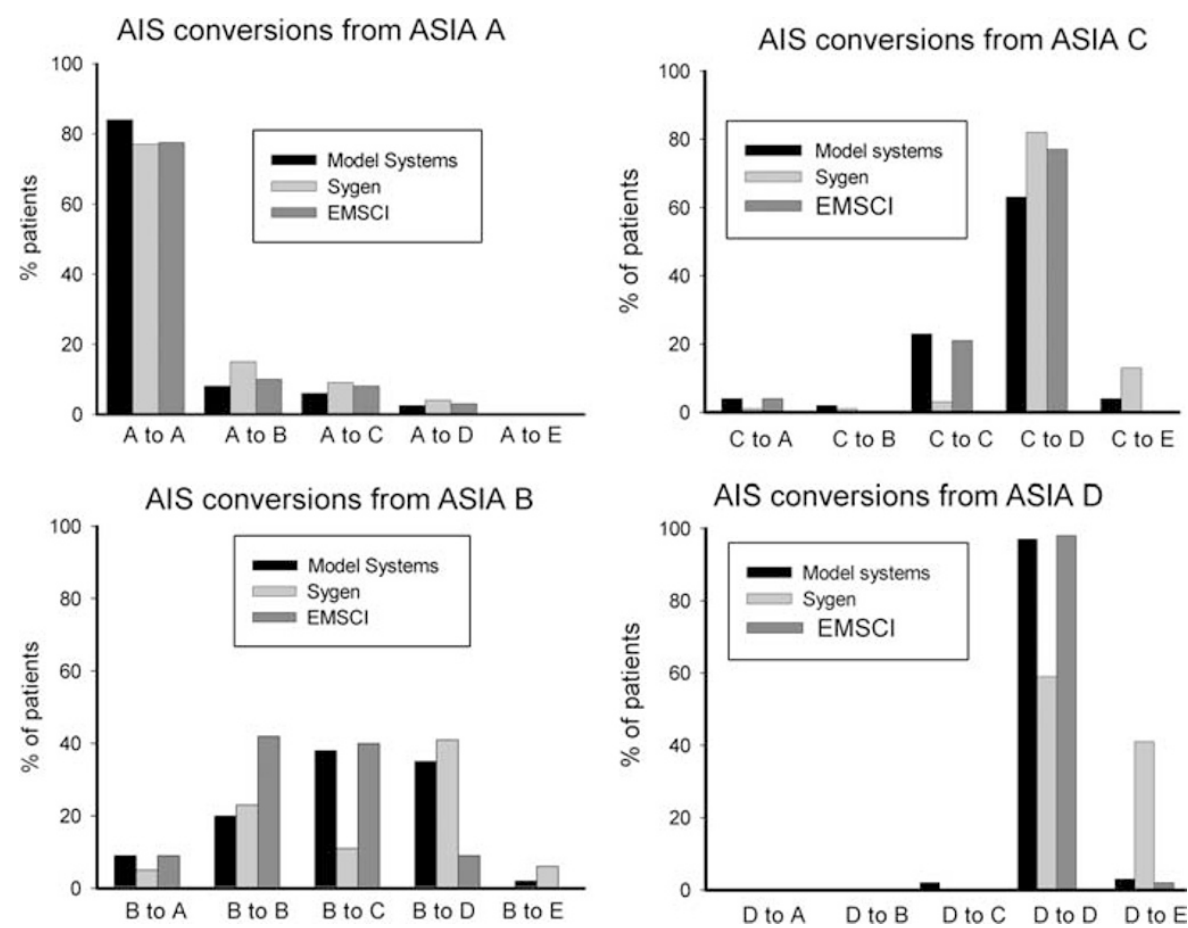

Figure 1 Percent AIS conversion from initial examination (within 3 days to 4 weeks of SCI) to the 1 year anniversary date after SCI. Data are from the US Model Systems, Sygen and EMSCI databases

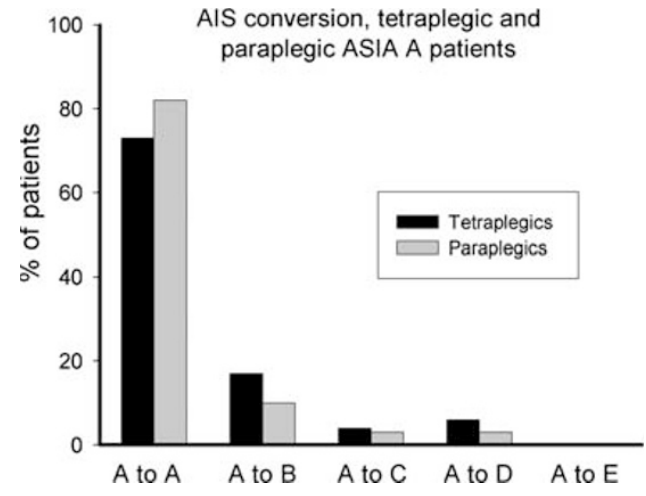

Figure 2 Comparison of percentage conversion of AIS Grade in AIS A tetraplegic and paraplegic patients from the EMSCI database at the 1 year anniversary date after SCI

Thus, in clinical trials involving AIS C or AIS D subjects, there is a potential 'ceiling effect' for the use of AIS grades where the assessment for the therapeutic activity or benefit of an intervention cannot be differentiated statistically from the neurologic improvement that is due to spontaneous recovery. Conversely, relying on the conversion from AIS A to another AIS grade as a primary clinical end-point may be too demanding a threshold for demonstrating therapeutic efficacy (ie potentially insensitive to all but the largest of treatment effects).

Therefore, it is unlikely that the conversion of AIS grades will be of value for assessing the potentially subtle beneficial effects of experimental SCI treatments in human clinical trials. Changes in ASIA motor scores or some functional outcome measure may be more useful readouts of therapeutic efficacy (see accompanying paper, Steeves $e t a l^{9}$ ). However, AIS grades can be of substantial value for the inclusion or exclusion of potential trial participants, as well as in the stratification of subjects into separate trial cohorts.

Time profile (stages) of recovery in the AIS

Over what time period does the change in neurologic or functional recovery after SCI occur? In Figure 3, we have plotted data provided from the EMSCI database, showing the time at which a change in AIS grade was noted in 67 of the 217 patients who showed any change, independent of SCI level (ie includes all subjects AIS A through AIS D). Approximately, $80 \%$ of these 67 patients showed a conversion within the first 3 months after injury. However, at later time points some AIS conversions were still observed.

Reanalysis of the Sygen data shows that, of 716 patients, 250 were able to show 'marked recovery,' meaning improvement of at least 2 grades in the Benzel Scale between the first examination at 2 days and 1 year. Of these 250, 62 were first able to meet this criterion at the 4-week-exam, 80 first met it at 8 weeks, 50 at 16 weeks, 38 at 26 weeks and 20 at 52 weeks. Thus, $57 \%$ of those who eventually reached this stringent standard of recovery had already done so within 2 months, and $77 \%$ had done so by 3 months. Some recovery did, however, continue throughout the observation period. A total of 


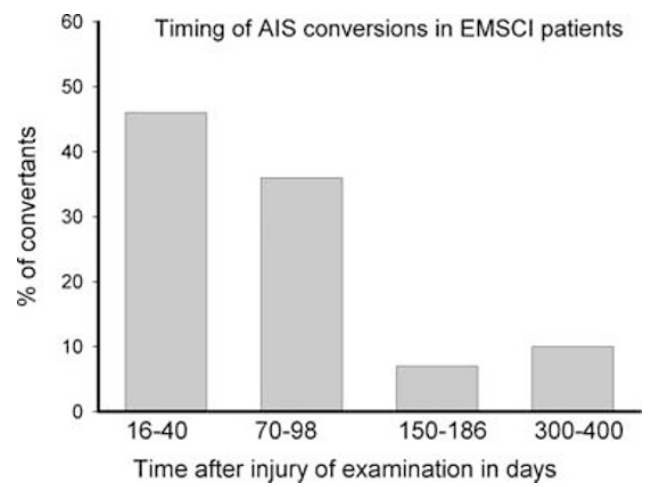

Figure 3 Timeframe at which patients in the EMSCI study showed an AIS grade conversion. A total of 67 subjects of 217 participants showed a conversion, independent of SCI level or severity

170 patients were able to improve by at least 1 AIS/ Benzel grade between baseline and 4 weeks, 148 did so between weeks 4 and 8, 132 improved between weeks 8 and 16, 109 between weeks 16 and 26, and 67 between weeks 26 and 52 .

A recent study by Kirshblum et $a l^{12}$ examined the degree of AIS conversion between 1 and 5 years after SCI in 987 subjects. They noted that $5.6 \%$ of people, who were neurologically complete (AIS A) 1 year after SCI, still converted to an incomplete injury by year 5 (ie over the next 4 years), with $3.5 \%$ converting to AIS B and about $1 \%$ to either AIS C or AIS D.

When does the spontaneous conversion of neurologic recovery begin to stabilize after SCI? This information is important in the design of clinical trial protocols, as well as for establishing appropriate outcome threshold values to demonstrate a statistically significant clinical benefit for any experimental intervention, including late rehabilitation strategies.

Depending on the therapeutic target SCI clinical trials will begin at various times after injury. It is somewhat of an arbitrary decision as to when you classify SCI as acute, subacute or chronic and likely to depend on the targeted pathophysiology or mode and mechanism of the therapeutic intervention being investigated. The Panel examined the changes for a number of different neurologic measures and functional capacities after SCI over time. We reviewed the variability of these values at any given timepoint, as well as the known neuropathological mechanisms and neuro-repair potential associated with these timepoints from a number of preclinical and clinical SCI studies. Whatever timepoint is chosen for the commencement of a clinical trial using an experimental treatment, it should be supported by appropriate preclinical data testing with a similar time frame (scaled for human studies).

There is little doubt that an experimental treatment administered within the first 3 days of SCI would be currently viewed as an acute treatment. How long the acute stage of human SCI persists is presently an open question. When exactly the chronic SCI state is achieved is another dilemma. Based on the available data, it might be suggested that the chronic state is only attained 12 months after SCI (where the preceding 6 months have indicated no change in functional capacity, thereby providing a stable baseline).

By default, all time points in between acute and chronic are classified as subacute, but in the case of human SCI, this could then be a time period almost as long as a year! Admittedly, these are far from rigorous (or satisfactory) timeframes, but there is little available evidence to support more rigid definitions until more precise information is available about any time limitations on the pathophysiology of human SCI. Functional recovery during the subacute period can be highly variable and the panel did not reach consensus on a further subdivision of this time period. Given the highly variable nature of the subacute state after SCI, investigators are cautioned that they may need to stratify trial cohorts, on the basis of time after SCI, to draw meaningful conclusions.

As, neurologic recovery after SCI is non-linear, especially within any subacute period, clinical investigators will wish to know the conversion rate from the beginning of their study, whenever that commences. For instance, if the study is to begin 8 weeks after SCI, the relevant data might be the changes in AIS grade between 8 weeks and 1 year after SCI. We have re-examined the Sygen database in order to provide this analysis. The AIS grade outcomes of patients in the Sygen database who were AIS A (which equals Benzel 1) at 3 days, are then plotted at 4, 8, 16, 26 and 52 weeks, with different plots for patients with cervical and thoracic injuries. Tables and graphs showing the full set of data are presented as Supplementary Figure 1. We present four graphs from this data set as Figure 4, to show that the change in Benzel grade between the beginning of a study starting at 3 days after SCI is much greater than the change observed when the study commences at 8 weeks after SCI (both trials concluding at 1 year after SCI). These new calculations and the EMSCI data show that a few patients who have previously shown no improvement can convert to a higher grade even months after injury. Even patients who are AIS A at 4 and 8 weeks after SCI can still show significant spontaneous improvement.

\section{Motor recovery after SCI}

Motor assessments were made in all the studies using the ASIA scale or an equivalent. In current research, the upper and lower extremity scores are usually given separately, but this was generally not the case in the studies we reviewed. As the ASIA motor assessment only covers five spinal levels in the cervical and lumbar regions, with no scores at the thoracic levels there are ceiling effects. Thus, a patient with a $\mathrm{C} 7$ injury could only be demonstrated to have two levels or 20 points of improvement down to T1. Any further improvement could not be scored because it would affect thoracic motor levels. All studies have reported an increase in 

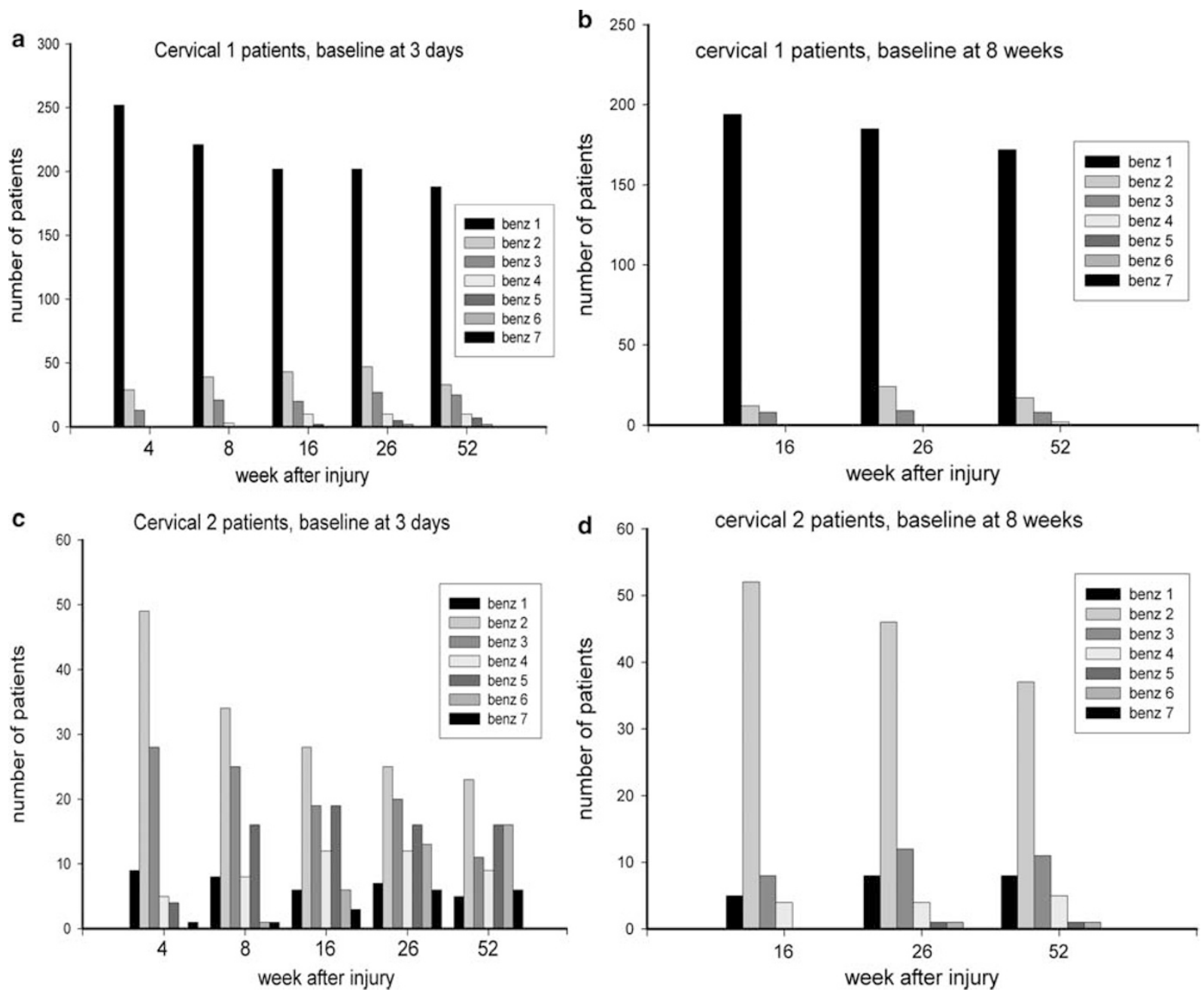

Figure 4 Benzel grades at various times after SCI calculated from the Sygen database. (a) shows the outcomes for subjects who were initially cervical 1 (which equates to cervical A or Benzel grade 1) when the baseline measurement started at 3 days, (b) shows the outcome pattern for subjects who were cervical 1 (which equates to Benzel 1 or AIS A) at 8 weeks. (c) and (d) show cervical 2 (equals cervical B or Benzel 2) subjects with the baseline starting at either 3 days and 8 weeks

ASIA motor scores during the first year after SCI. The numbers of motor points gained during the first year are rather consistent between studies. As with conversion of AIS grades, incomplete SCI patients show greater degrees of motor recovery than AIS A patients. The overall increases found in four studies are shown graphically as Figure 5. The recovery rates in the AIS $\mathrm{C}$ and D group are probably greater than shown in this graph, but there is a ceiling to the increase that can be shown in the ASIA motor score, as described above.

The Sygen database also contains data on thoracic level SCI. Thoracic AIS A patients showed a mean increase of 4.5 motor points over the first year, and thoracic B patients showed a 22.7 point increase. The presentation of the Sygen data in Geisler et $a l^{6}$ did not include statistical analysis, making it impossible to perform power calculations. However, these statistics are now presented in Supplementary Figure 2.

Motor recovery over the first year after SCI was also examined in the NASCIS II trial control group. ${ }^{3}$ It should be remembered that given the time between the NASCIS study and the more recent adoption of the ASIA Impairment Scale there are differences between the motor scoring systems which means that the data cannot be compared directly. ASIA utilizes 10 muscles on each side of the body for a maximum total score $(20 \times 5)$ of 100 ; whereas NASCIS used 14 muscles on only one side of the body for a maximum total score of 70. The following data is for 43 subjects in which the initial ASIA assessment was made within $8 \mathrm{~h}$. The observed motor recovery after 1 year was 4.6 points for AIS A subjects, 31.3 points for AIS B subjects, and 12.9 motor points for AIS C and D subjects combined.

\section{Motor recovery at intermediate time points}

In many of the published studies, ASIA motor examinations were made at various times after SCI until the end of the study 1 year after SCI. For example, the study by Waters et al ${ }^{10}$ followed the outcome of 61 

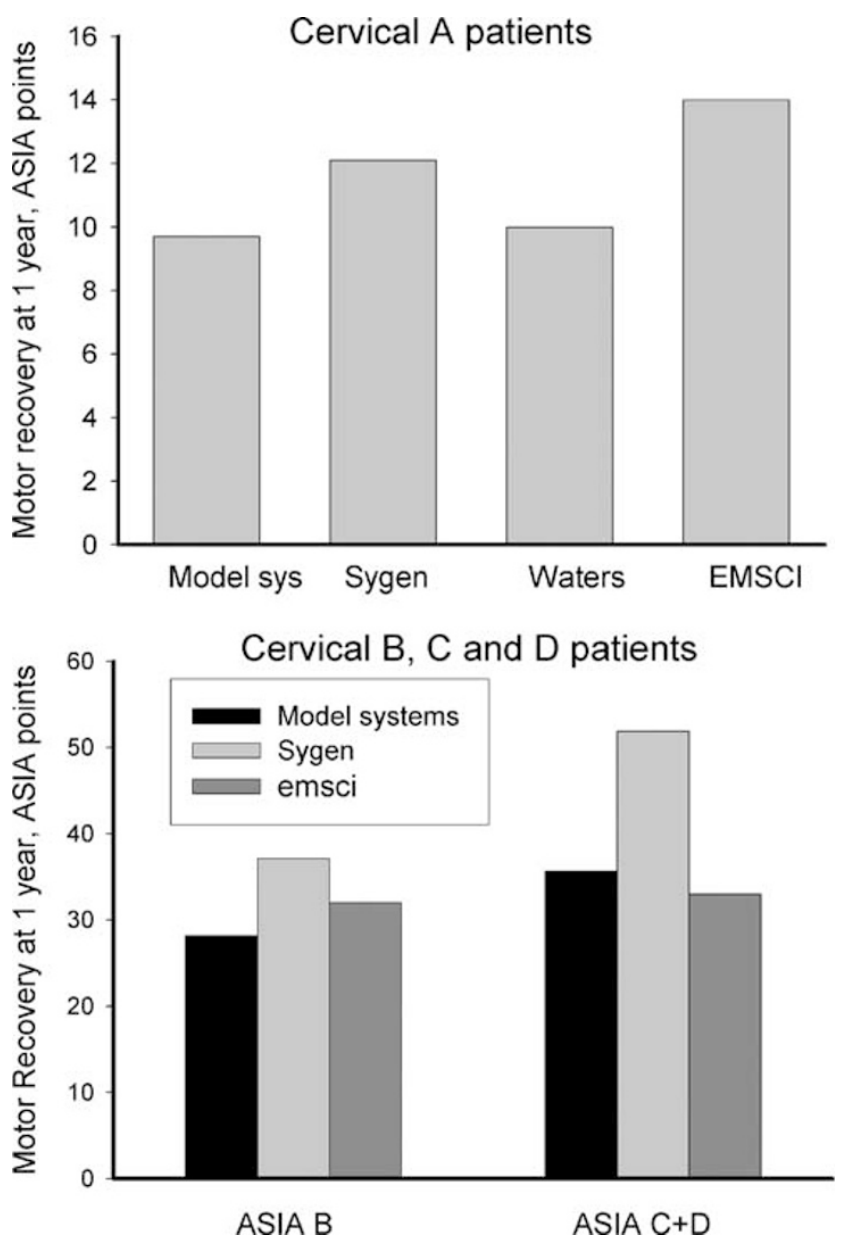

Figure 5 Motor recovery, measured in ASIA motor points, over the first year after SCI in AIS A, B and C/D patients. The rates of recovery in the Model systems, Sygen and EMSCI studies are compared

complete tetraplegic patients with lesions between $\mathrm{C} 4$ and $\mathrm{C} 7$. The patients were first examined at admission to a rehabilitation spinal injury unit (within 30 days of $\mathrm{SCI}$ ). The cumulative motor recovery of these patients is shown in Figure 6. In short, cervical-injured AIS A patients showed a mean improvement in motor score of 10 by the end of 1 year after SCI and this only improved to 11 by the end of the second year after SCI.

The most complete data come from the Sygen study. We have reanalysed the Sygen data, and now provide information that is not in the original paper. First, we show the standard deviations for the mean improvement in motor score over the 1 year assessment period of the study (Supplementary Figure 2). Second, in order to assist those investigators designing studies which do not begin immediately after SCI, we have recalculated the rates of motor recovery, assuming that subjects are randomised, assigned an AIS grade and admitted to the study at various time points during the first year after SCI. We have calculated changes in motor scores from participants who were AIS A or AIS B starting at 4,8 , 16 and 26 weeks after SCI, with all assessments

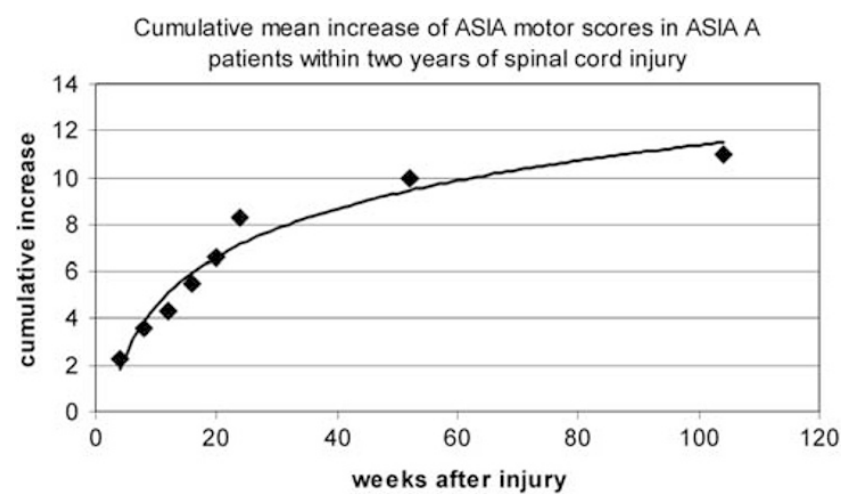

Figure 6 Change in ASIA motor score for sensorimotor complete (AIS A) patients within initial two years after SCI (Waters et al. ${ }^{10}$ ). Note that most rapid improvement occurs within the first 6 months after SCI and is essentially maximal after 12 months

concluding at 52 weeks after SCI. This new presentation provides some of the necessary data needed to perform power calculations for trials that might begin with an experimental intervention at different time points after SCI. These power calculations appear in a later section of this paper. The full set of tables is presented as Supplementary Figure 2. We show representative plots here (Figure 7) where we have plotted recovery curves from some of the Sygen motor recovery data.

\section{Rate of motor recovery}

All studies give fairly consistent data on the rate and timing of functional recovery. The rate of recovery is rapid during the first three months and motor improvement is almost complete by 9 months, but ultimately only plateaus at 12 to 18 months after SCI. This can be seen from the motor recovery graphs in Figures 7 and 8. These figures also show that the rate and extent of recovery is greater in patients with incomplete lesions. Rate of motor recovery was examined explicitly in several studies. For example, Figure 8 shows a graph from a review by Burns and Ditunno ${ }^{13}$ which presents a typical picture and is taken from previous data. ${ }^{14}$

Ditunno et $a l^{15}$ examined the rate of motor recovery in a different way, by examining motor recovery in the right biceps of motor complete (AIS A and AIS B) and incomplete (AIS C and AIS D) SCI subjects, classified as $\mathrm{C} 4$ right motor level. The biceps is primarily supplied by motor innervation from the immediately adjacent C5 spinal level. The results from 40 patients, 27 motor complete and 13 incomplete show progressive recovery, fastest over the first 3 months, with a large proportion of patients $(70 \%$ of complete SCI and $90 \%$ of incomplete SCI subjects) showing recovery from a grade of either $0 /$ 5 (complete) or $2 / 5$ (incomplete) to a useful motor grade (3/5 or better) within 12 months of the initial injury.

Motor recovery in the segments close below the lesion It is probable that several of the prospective treatments 

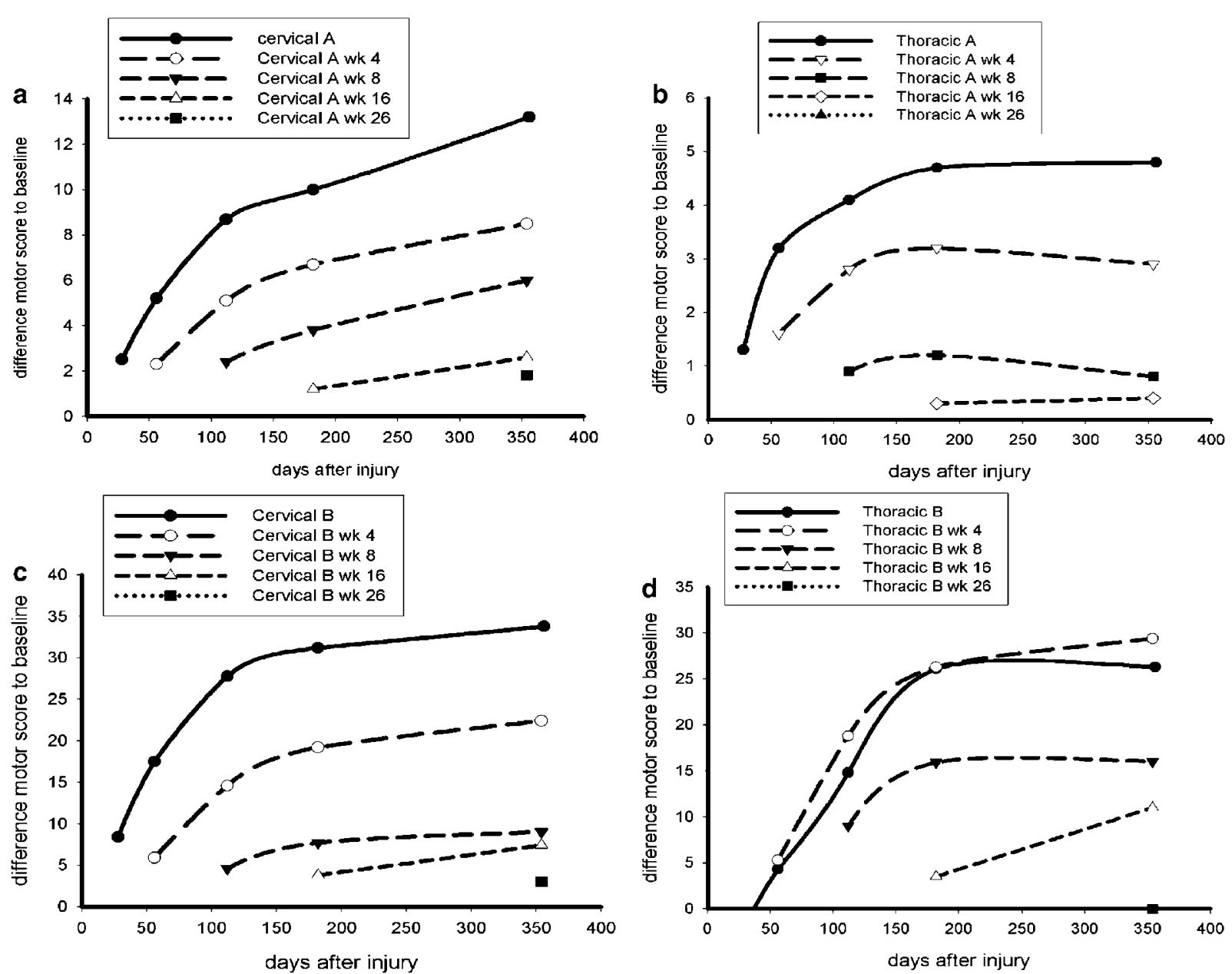

Figure 7 Motor recovery of Cervical AIS A (a), thoracic AIS A (b), cervical AIS B (c), thoracic AIS B (d) patients, calculated from the Sygen database, using the placebo-group data. The solid lines show recovery from the beginning of the study, as in Geisler et al. ${ }^{6,7}$ In order to demonstrate expected recovery rates for studies beginning at 4, 8, 16 or 26 weeks after SCI, recovery rates are shown for if the study were to start at such delayed treatment times. The data are therefore not confounded by patients who converted to a different AIS grade between day 3 and a delayed start time of 4, 8, 16 or 26 weeks after SCI

that will undergo SCI trials will have their maximum effect within spinal levels just below the injury. There is therefore, a need for data on spontaneous recovery rates in these spinal segments. The issue is complicated by the existence of a zone of partial preservation (ZPP) in most patients with complete injuries, where some motor capacity remains. Moreover, this zone varies in size from patient to patient. Spinal levels below the ASIA motor level where there is some measurable muscle function clearly lie within the zone of partial preservation. Of particular interest for clinical trials may be the first or second motor level at which there is zero function. However, if the initial neurological assessment was performed early, there is often some significant spontaneous motor recovery within these segments, which might therefore be considered to be within the ZPP. In principle recovery within the ZPP could be due to both CNS and peripheral plasticity, while recovery beyond the ZPP would probably require at least some CNS repair (eg axon regeneration).
Changes in function versus distance below the lesion A small retrospective study from Vancouver ${ }^{16}$ examined return of motor function at adjacent distal levels below the neurological level as determined using the ASIA motor scores. Figure 9 is a graph (based on the data in Table 4 from the paper), which shows the number of tetraplegic patients that either do or do not recover useful motor function (grade $3 / 5$ or better) after 2 or more years following SCI. The results emphasize that most motor recovery occurs within the first level below the ASIA motor level $(100 \%$ for incomplete tetraplegic SCI and $56 \%$ for complete tetraplegics). Furthermore, there is very little or no recovery at more than two spinal levels below the initial ASIA level.

Waters et $a l^{10}$ also examined recovery of function in spinal levels below the injury level for complete (AIS A) tetraplegic patients. The observations at 1 year, based on initial assessment within 30 days of SCI, were that $57 \%$ of the 88 cases showed improvement from $0 / 5$ to $1 / 5$ strength in the immediately adjacent level (first zero- 


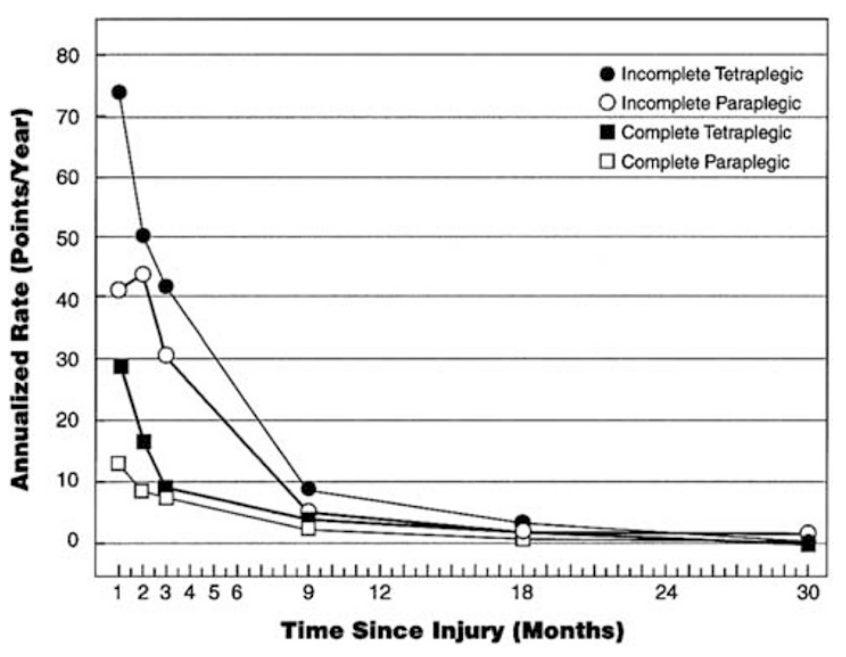

Figure 8 Motor recovery for complete and incomplete spinal cord injury patients over the first 2.5 years after SCI (Waters ${ }^{14}$; Burns and Ditunno ${ }^{13}$ ). Note the most dramatic improvements occur over the first 9 months, although a completely stable plateau (baseline) may not be achieved until 18 months after SCI, depending on the severity and level of the injury (incomplete tetraplegia taking the longest to stabilize)

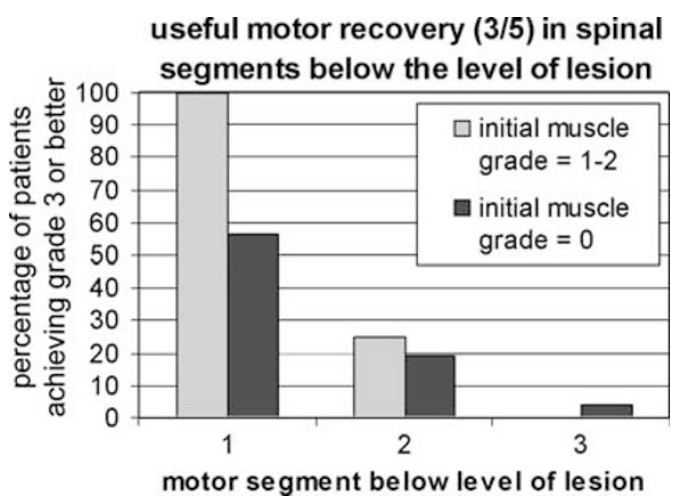

Figure 9 Recovery of useful motor function in spinal segments below the ASIA motor level (Fisher et $a l^{16}$ ). The figure shows the percentage of patients who initially either had no motor function (muscle grade $=0$ ) or minimal muscle strength (grade 1-2) who subsequently recovered some useful function at each spinal level below the initially defined level of motor lesion

rated) 1 year after injury, while only $27 \%$ improved to $3 / 5$ or better (ie useful motor function). In the second adjacent motor segment below the level of lesion, only $4 \%$ of patients showed any measurable strength $(1 / 5)$, and only $1 \%$ (one patient) regained useful motor recovery ( $3 / 5$ or better).

There is some evidence that the motor grade of the lowest functional level affects the probability that function will return in the level below it, although this is based on groups of only 20 or so patients with $\mathrm{C} 5$ and C6 injuries. Burns and Ditunno ${ }^{13}$ reviewed the probability of useful motor recovery (to grade 3 or more) one level below a level with some detectable function. If the segment above was initially graded 2.5 out of $5,100 \%$ of patients regained function to a grade of 3 in the next segment below. If the segment above was initially only rated a grade of $1-2.5$ out of 5 , then $75-80 \%$ of patients regained grade 3 or better function in the next segment below. However, if the segment above was initially assessed a grade of 0 , then only $25-30 \%$ of patients regained function to a grade of 3 in the next segment below.

The timing and extent of motor recovery within the ZPP was examined by Ditunno et al. ${ }^{17}$ They examined the time course of recovery in muscles with no voluntary movement (grade 0) compared with muscles with some preserved motor function (grades 1 or 2), but less than a functional (antigravity) grade 3 muscle level. Initial ASIA assessment was within one week of SCI. Figure 10 shows the percent of patients achieving grade 3 or better in impaired muscles, showing higher levels of recovery in muscles that were grade 1-2 compared with grade 0 . The conclusion from this study, and others from this research group, is that recovery in muscles with some voluntary function is both faster and more complete than in muscles that initially have no function. The great majority of improvement appears to occur within the first 9 months after SCI.

\section{Sensory recovery after SCI}

Sensory function and changes in sensory level are often described as changes in ASIA sensory score after injury. However, in many studies, recovery of sensory function was not taken as a primary outcome measure. This was because ASIA sensory examination often provided somewhat variable results within individual patients. In addition, the sensitivity of the ASIA sensory score is limited with only three grades of sensation being scored for detecting a light touch or pin prick stimulus, where $0=$ absent, $1=$ impaired and $2=$ normal in the affected dermatome.

\section{Data from the Sygen study}

The rates of sensory recovery in patients from the Sygen study starting at the beginning of the study are shown in Figure 11, and the statistics are in Supplementary Figure 3 . We have also made new calculations, as with the motor data shown in Figure 7. In these, we have provided recovery data relevant to trials that might be started at later time points. We have taken the patients who were Cervical A or B and Thoracic A or B and then show the subsequent sensory score recovery at $4,8,16$, 26 and 52 weeks, out of a maximum of 112 (26 dermatomes on left and 26 on right side with a maximum score of 2 for each dermatome), and we have provided recovery data for patients graded AIS A and B at 4, 8, 16 and 26 weeks. The complete data are provided in Supplementary Figure 3.

It has been suggested that preservation of pinprick sensation may predict motor recovery in persons with motor complete, sensory incomplete (AIS B) SCI. 
Recently, the Sygen data was used to re-examine this possibility. ${ }^{18}$ The retrospective examination of 131 AIS $B$ patients indicated that a higher percentage of individuals with sacral sparing of pinprick sensation (within $72 \mathrm{~h}$ of SCI) were ambulating at 26 or 52 weeks after SCI, although this did not reach statistical significance. However, preservation of sacral pinprick at 4 weeks after SCI was significant for predicting ambulation at 1 year after SCI. If lower extremity (L2$\mathrm{S} 1$ dermatomes) pinprick sensation was maintained in $50 \%$ or more of the dermatomes then a significant proportion of patients would recover ambulatory function. Thus, the preservation of pinprick sensation appears to be useful in predicting motor recovery. ${ }^{18}$

In Table 1, we show the sensory recovery data from Waters et $a l^{10}$ for a subset of 55 AIS A tetraplegic

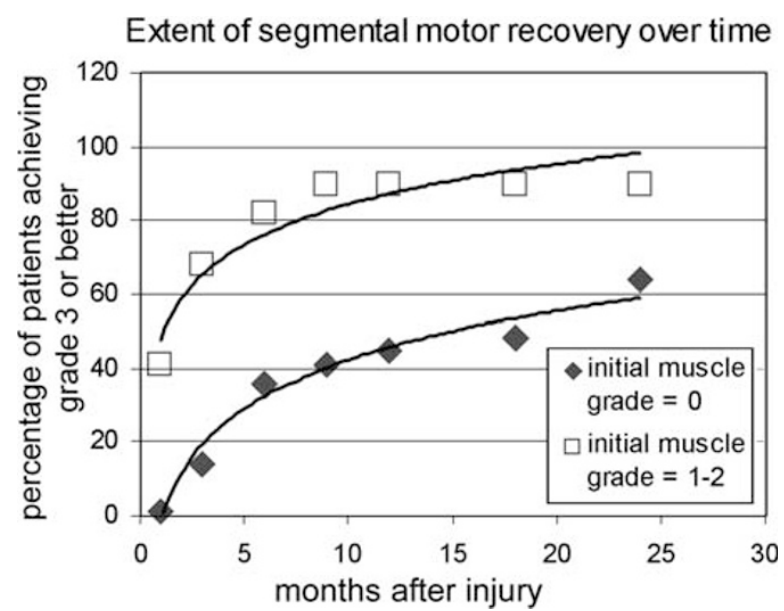

Figure 10 Motor recovery over time within the zone of partial preservation (redrawn from data in Ditunno et $a l^{17}$ ) subjects who remained complete from a set of 61 subjects classified as complete at admission. The apparent degree of recovery is more modest than that obtained from the Sygen sensory assessments.

\section{Data from the NASCIS study}

The scale for the NASCIS sensory scores is rather different from that used in the Sygen study. The same dermatomes are scored but unilaterally, and assigned a three point score from 1 to 3 instead of 0 to 2 . The increases in sensory scores from baseline from the two sets of data can therefore be compared by doubling the values from the NASCIS data set. The recovery of sensory scores after 1 year in 43 placebo-treated complete and incomplete SCI subjects (with baseline assessment within $8 \mathrm{~h}$ of SCI) for AIS A subjects were 5.5 points for light touch and 5.1 points for pinprick sensation. Likewise, for AIS B participants, the improvement in sensory scores were 10.8 for light touch and 15.8 for pinprick; whereas for combined data from AIS C and D subjects indicate a possible 'ceiling effect' as the improvements were only 3.0 for light touch and 9.2 for pinprick.

\section{Prognostic value of the ASIA examination at early assessment times}

For a neuroprotective treatment, the sooner the treatment is applied, the more likely it will be effective. Thus, another key issue will be when a reliable baseline ASIA classification can be established for a person with SCI. For numerous reasons, such as spinal shock, medical instability, concomitant brain injury or coma, the ASIA assessment within the first $24 \mathrm{~h}$ of SCI can be inherently
Recovery of sensory function, pinprick

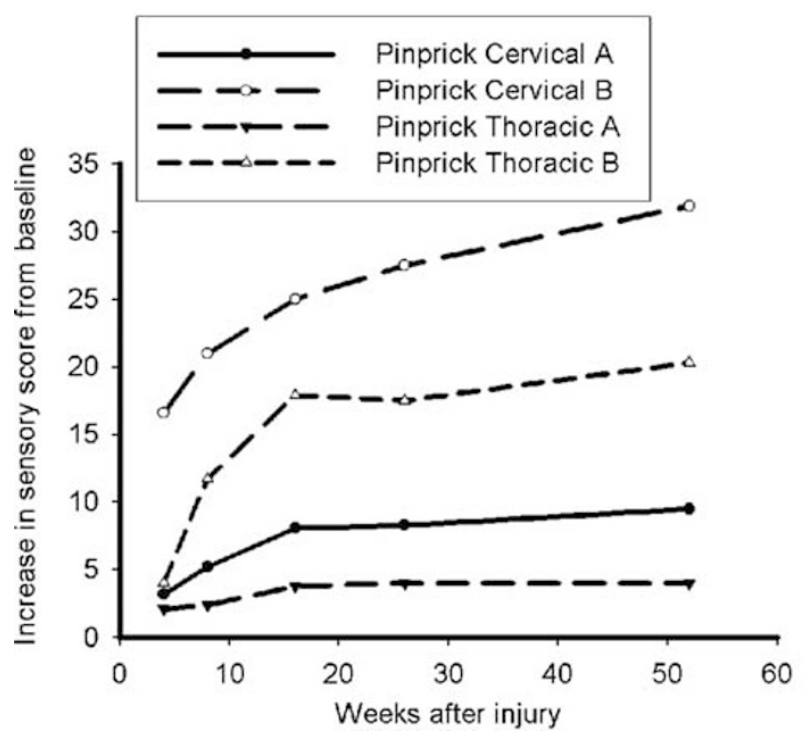

Recovery of sensory function, light touch

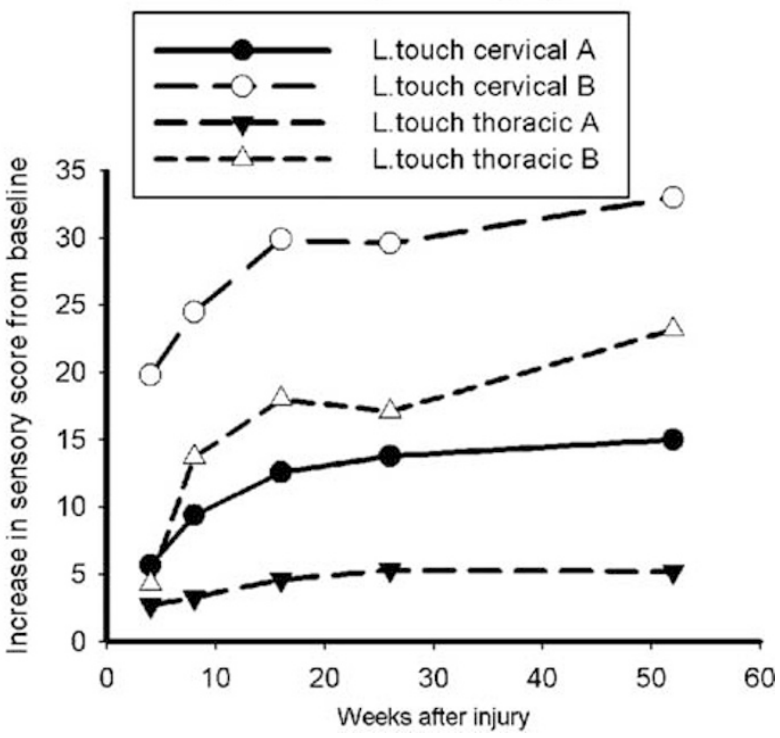

Figure 11 Sensory recovery data from the Sygen study. The cumulative increases in light touch and pinprick sensory scores from a baseline of 3 days after SCI are shown 
Table 1 Mean ASIA sensory score changes for tetraplegic ASIA A subjects, relative to a baseline assessment one month after SCI (from Waters et $a l^{10}$ )

\begin{tabular}{lcccccccc}
\hline Re-examination at & 2 month & 3 month & 4 month & 5 month & 6 month & 1 year & 2 year & 3 year \\
\hline$n$ (\# subjects) & 55 & 55 & 55 & 47 & 37 & 34 & 20 & 12 \\
Light touch & 0.7 & 1.2 & 1.3 & 1.6 & 1.7 & 2.3 & 2.5 & 2.6 \\
Sharp touch & 0.7 & 0.7 & 1.0 & 1.3 & 1.5 & 1.9 & 2.2 & 2.7 \\
\hline
\end{tabular}

unreliable as a predictor of the subject's future functional disability or capacity. Assessment at $72 \mathrm{~h}$ after SCI is generally accepted as having a more accurate prognostic value.

This issue was examined specifically by Brown et al ${ }^{19}$ Motor complete (AIS A and AIS B) patients $(n=29)$ were examined within $24 \mathrm{~h}$ of SCI, then again at $72 \mathrm{~h}$ and at several times up to 3 months after SCI to determine how well the motor exam at each time point predicted subsequent functional recovery (to muscle strength grades of 4 or 5) at 3 months. The study examined the strongest muscle within the ZPP. Between 24 and $72 \mathrm{~h}, 47 \%$ of patients with a grade 3 strength in the selected muscle showed a decline in strength to grade 1 or 2 , while $17 \%$ of those with grade 1-2 increased to grade 3 . A total of $71 \%$ of the subjects with a grade 3 strength in the selected muscle at $24 \mathrm{~h}$ showed recovery to grade 4 or 5 , compared to $33 \%$ of those with grade $1-2$, a difference that was not statistically significant. However, all of the 11 subjects with a grade 3 muscle strength at $72 \mathrm{~h}$ showed recovery to grade 4 or 5 by 3 months, while only $28 \%$ of those with a grade of 1 or 2 recovered to grade 3 or above, which was a significant difference. The conclusion is that the $72 \mathrm{~h}$ examination provides a better prediction of outcome in motor complete (AIS A and B) patients, while the $24 \mathrm{~h}$ examination is not as reliable. It may be noteworthy that the primary difference in this small study appeared to be due to those subjects whose strength declined between 24 and $72 \mathrm{~h}$ and did not subsequently recover.

The reliability of the initial and second examination in motor complete patients was also examined by Burns et al. ${ }^{20}$ Patients were examined initially within $48 \mathrm{~h}$ of SCI, again within a week before discharge and then 1 year after SCI. The early examination was classified as unreliable if there were factors affecting cognition (eg, traumatic brain injury, drug effects, psychological disorders), communication (patient on ventilator, language barriers) or other considerations that would undermine confidence in the examination. As can be seen from the data below (Table 2), there was a higher rate of apparent recovery in the patients with an unreliable initial $(<48 \mathrm{~h})$ examination. The conclusion was that a reliable initial examination may be as reliable as a second, later examination.

From these data, it is clear that it is desirable to perform a detailed neurologic examination and ASIA assessment just before randomization of patients into trial groups and as close as possible to the initiation of treatment. For trials where the treatment is to be given within the first 3 days after SCI, it may be prudent to exclude patients where the clinical examination may not provide reliable information.

\section{Number of subjects for valid statistically significant SCI clinical trials}

A key consideration in trial design is the number of patients that need to be recruited and enrolled in a clinical trial in order to achieve a significant result. We have reanalysed the control arm data from the Sygen study ${ }^{6}$ in order to calculate the number of patients in each arm of the study required to show a statistically significant difference between an experimental and control group for a RCT of an experimental treatment for SCI. We have used the whole Sygen database for the calculations shown in Figure 12 because the placebo group is too small to provide reliable figures; all the calculations are shown in Supplementary Figure 4. These calculations are based solely on the ASIA motor score, and trials designed using more sophisticated, more accurate or composite outcome assessments may require fewer numbers. However, these calculations provide an indication of the number of subjects that might be required in a study and how the magnitude of spontaneous recovery can influence those numbers.

We have used two assumed treatment effects, a difference of either 5 or 10 points in the ASIA motor subscore between the experimental and control groups. These are relatively modest treatment effects, and trial group sizes would be considerably smaller if the designated clinical end point motor score was a larger difference (eg 20 point difference in ASIA motor scores). As the trials of different treatments might begin at different times, and because functional capabilities become less variable with time after injury, we have made calculations assuming a clinical trial might begin at various times after SCI. For instance, a trial of a neuroprotective substance might begin as soon after a spinal injury as possible, while a trial of a regeneration/ repair-inducing treatment might begin within 1-4 weeks, and, finally, a trial of a neural plasticity-inducing intervention might begin at almost any time, including during the chronic state of SCI (eg >12 months after injury). A graphical representation of these results is shown in Figure 12.

For example, if the SCI trial were confined to Cervical AIS A patients enrolled immediately after SCI (eg a 
Table 2 Reliability of the ASIA examination within $48 \mathrm{~h}$ after SCI. ASIA assessments that were thought to be unreliable resulted in a higher number of subsequent ASIA grade conversions (Burns et $a l^{20}$ )

\begin{tabular}{|c|c|c|c|c|c|}
\hline \multirow{2}{*}{$\frac{\text { ASIA Examination Reliability }}{\text { Initial Admission ASIA grade }(<48 \mathrm{~h})}$} & \multirow[t]{2}{*}{$\begin{array}{l}\text { Assessed } \\
\text { Asia Grade }\end{array}$} & \multicolumn{2}{|c|}{ Thought to be Reliable } & \multicolumn{2}{|c|}{ Thought to be Unreliable } \\
\hline & & $\mathrm{A}(n=38)$ & $\mathrm{B}(n=13)$ & $\mathrm{A}(n=43)$ & $\mathrm{B}(n=9)$ \\
\hline Subsequent ASIA Grade at second & A & 37 & 0 & 39 & 0 \\
\hline exam (usually within one week of initial & $\mathrm{B}$ & 1 & 10 & 4 & 9 \\
\hline \multirow{2}{*}{ admission exam; $n=103$ ) } & $\mathrm{C}$ & 0 & 2 & 0 & 0 \\
\hline & $\mathrm{D}$ & 0 & 1 & 0 & 0 \\
\hline Initial Admission ASIA grade $(<48 \mathrm{~h})$ & & $\mathrm{A}(n=30)$ & $\mathrm{B}(n=10)$ & $\mathrm{A}(n=23)$ & $\mathrm{B}(n=5)$ \\
\hline Subsequent ASIA Grade at one year or & A & 28 & 0 & 19 & 0 \\
\hline \multirow[t]{3}{*}{ later $(n=68)$} & $\mathrm{B}$ & 2 & 4 & 1 & 2 \\
\hline & $\mathrm{C}$ & 0 & 3 & 2 & 3 \\
\hline & $\mathrm{D}$ & 0 & 3 & 1 & 0 \\
\hline
\end{tabular}

Number of subjects for valid statistically-significant SCI clinical trials

neuroprotective treatment) and powered to show a statistically significant difference of 10 ASIA motor points between the experimental and control groups, it would be necessary to enrol approximately 60 subjects for each arm of the study, but over 230 subjects if the difference was reduced to five motor points. However, for AIS B cervical-injured candidates in a similar trial design the numbers increase dramatically to 277 and 1105, respectively, because of the magnitude and variability of motor recovery. Fortunately, because the rate of spontaneous recovery decreases with time after SCI, the number of patients that would have to be recruited into a trial becomes smaller as the initiation of treatment is delayed. The full set of tables for these calculations is presented in Supplementary Figure 4. The sample size (power) calculations produce very similar results when performed using the data from the placebocontrol group alone or with the combined data from both the experimental and control arms of the Sygen study.

\section{Summary}

Almost all people with SCI show some recovery of motor function below the initial ASIA injury level. In patients with motor complete lesions (AIS A and AIS B) the majority of this functional return is likely to occur within the ZPP. Thus, a muscle group that has some degree of minimal function early after SCI has a higher probability of regaining some behaviourally useful function (muscle grade 3 or higher) with time. Recovery also appears to be particularly probable in myotomes with sensory preservation. This recovery may be sufficient to reclassify the ASIA injury level to a lower spinal level. The vast majority of this recovery occurs in the first 3 months, but a small amount of ongoing recovery can persist for up to 18 months or occasionally longer.

It is not clear from most previous studies how much of the motor recovery is seen below the ZPP. In some of the studies there was recovery in some zero-rated muscles, but only if they were one segment below the ZPP. Recovery two segments below the most caudal segment of the ZPP rarely occurs.

Some sensory recovery occurs after SCI, on roughly the same time course as motor recovery. The magnitude for the recovery of sensory function may appear to be less than or more variable than motor recovery, as the limited (3 point) scale for the ASIA sensory assessment and the need to rely on a subject's perception makes it less sensitive (more variable) than ASIA motor assessments.

While the spontaneous recovery of motor function in people with motor-complete SCI is fairly limited and predictable, recovery in incomplete SCI patients (AIS C and AIS D) is both more substantial and highly variable. Therefore, relatively smaller trial sample sizes may be possible when undertaking a trial with subjects having AIS A or AIS B SCI, but trials involving incomplete SCI patients, or trials where an accurate assessment of AIS grade cannot be made prior to the start of the trial (eg early application of a neuroprotective agent), will require larger numbers of subjects, randomized to an experimental or placebo-control study arm.

\section{Recommendations}

The data presented in the current literature come from assessment methodologies that were designed mainly to aid the prediction of outcomes for the purposes of rehabilitation and long-term care. For future clinical trials further forms of assessment will be used, as discussed in the second document in this series (Steeves et $\left.a l^{9}\right)$. For trials of regeneration/repair or plasticityinducing interventions, we believe that clinical motor data might be more useful if presented in a different form. This is because these recovery processes are likely to affect segments near the lesion, with a declining effect further from the lesion. It will be important to document recovery relative to the number of levels below the lesion, and whether recovery occurred within or beyond 

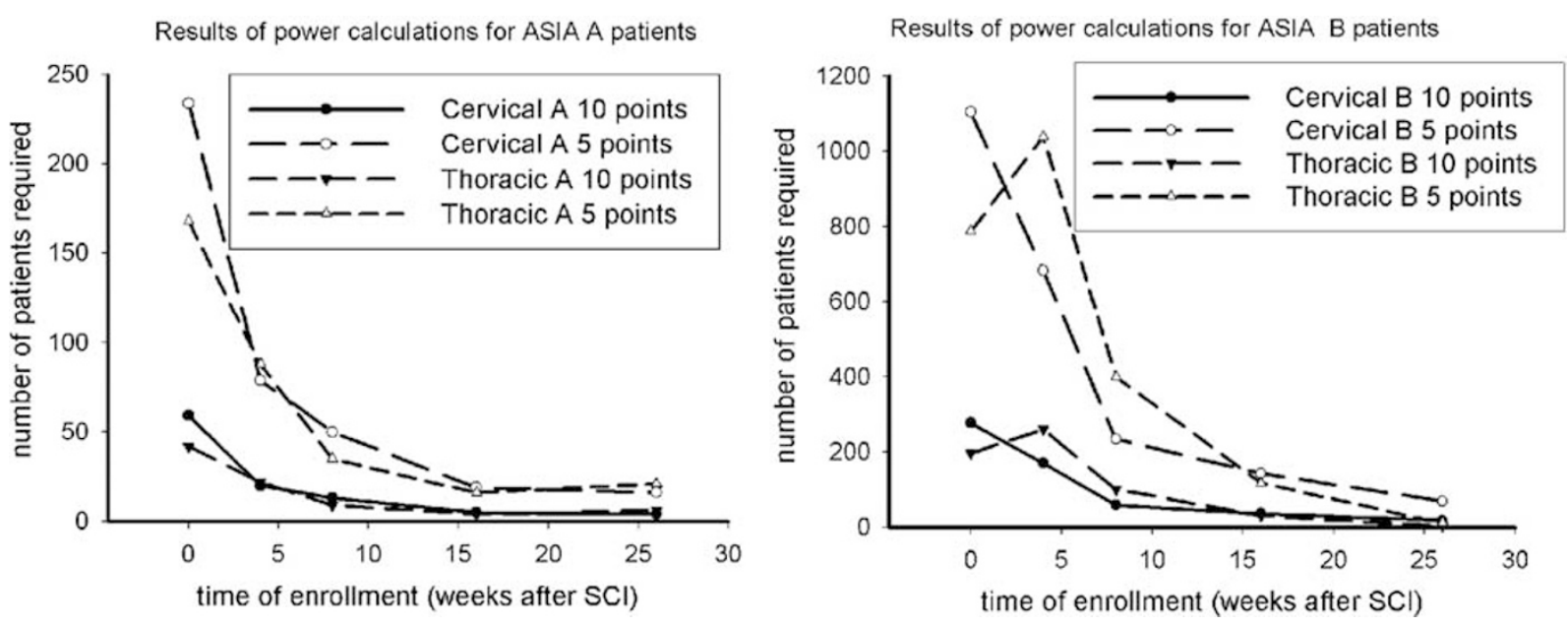

Figure 12 Graphs result from a re-analysis of the Sygen database, using combined data from both placebo and treatment groups. They show the projected number of patients/subjects per treatment arm that would have to be recruited into a clinical trial were the desired significance level $=0.05$. Calculations were performed on the basis of a treatment effect having a difference of 5 or 10 ASIA motor points between the experimental treatment group and the control group

the ZPP. Such data would also provide important information for understanding the mechanism of action for the development of subsequent generations of therapeutics. It will therefore, be useful to have patient data presented in terms of segments below the accurately described neurological ASIA level. It will also be important to map the ZPP for each subject, to correlate this with the ASIA lesion level, as well as that defined by imaging (CT or MRI) and to differentiate between recovery of function within the ZPP and recovery beyond this zone, as this more likely to be repair of spinal origin.

The panel therefore, decided that it will be useful to perform additional analyses of the major existing databases in order to provide two types of information that are not present in the current literature. In view of the likelihood that recovery of function following treatments will be concentrated close to the level of injury, it would be very useful to present some of the existing trial data in a format which shows recovery of function relative to distance below the neurologic level of injury. A reanalysis of the Sygen database and possibly others will be made for this purpose.

The numbers of patients required to demonstrate a significant difference between an experimental and control arm of a clinical trial will be relatively large if the trial begins soon after injury and if the clinical endpoint relies solely on differences in AIS grades or motor scores, as in most previous SCI trials. In order that SCI clinical trials can be undertaken in a more effective manner (with a manageable number of subjects) and within a reasonable time frame, alternative methods for analysing motor, sensory and autonomic function need to be validated as clinical outcome tools. These approaches are discussed in the second paper of this series.

\section{Glossary of definitions}

(Additional glossaries are included in the three accompanying papers)

A booklet and training manual is available from the ASIA that summarizes the AIS scale and clinical assessment protocol.

(http://www.asia-spinalinjury.org/publications/index. html)

Neurological Level of spinal injury is generally the lowest segment of the spinal cord with normal sensory and motor function on both sides of the body. However, the spinal level at which normal function is found often differs on each side of the body, as well as in terms of preserved sensory and motor function. Thus, up to four different segments may be identified in determining the neurological level and each of these segments is recorded separately and a single level descriptor is not used. Please note that the level of spinal column injury may not correlate with the neurological level of SCI.

ASIA (American Spinal Injury Association) Impairment Scale (or AIS) describes the completeness of a spinal injury. An individual with an AIS A grade has no motor or sensory function at the level of S4-S5 sacral segments. AIS B has some sensory function below the neurological level, including S4-5, but not motor function. AIS $\mathrm{C}$ has some motor function below the neurological level, but more than half of the key muscles involved have a muscle strength score which is $<3$ (Figure 1). AIS D has motor function below the neurological level but more than half of the key muscles have a muscle grade of 3 or more. AIS E indicates normal motor and sensory function.

Tetraplegia (quadriplegia) is the term used to refer to loss of motor and/or sensory function due to damage to the spinal cord, with impairment of the upper 
extremities as well as trunk, legs and pelvic organs. This implies damage to the spinal cord at or above the $\mathrm{C} 8$ level.

Paraplegia is the equivalent term used to refer to functional loss below the level of the upper extremities, which may involve loss of motor and/or sensory function within the trunk, and/or the lower extremities. This implies damage to the spinal cord below the level of C8 and may include damage to conus medullaris or cauda equine (ie neural tissue within the spinal canal).

Complete and incomplete SCI are other terms used to describe the overall severity of SCI. Technically, SCI is classified as complete if there is no motor or sensory function preservation in the sacral (most caudal) spinal segments. Thus, incomplete SCI is where there is some preserved motor or sensory function at the lowest sacral spinal level (S4/5). There can be extensive variability in the degree of preserved function after incomplete SCI.

ASIA sensory and motor assessments form the basis for the International Standards for Neurological and Functional Classification of SCI (the ASIA International Standards) and are conducted in the supine position and involve a qualitative grading of sensory responses to touch and pinprick at each of 28 dermatomes along each side of the body and a qualitative grading of the strength of contraction within 10 representative (key) muscles, primarily identified with a specific spinal level, 5 for the upper extremity (C5-T1) and 5 for the lower extremity (L2-S1) on each side of the body (Table 1).

ASIA motor score is calculated by assigning to one muscle group, innervated and primarily identified with a specific spinal level, a score between 0 (no detectable contraction) and 5 (active movement and a full range of movement against maximum resistance). C5-T1 and L2-S1 are tested, giving 10 levels on each side of the body for a possible maximum score of 100 .

LEMS is the lower extremity motor score which is a maximal 50 point subset of the ASIA motor score for the representative leg and foot muscles.

UEMS is the upper extremity motor score which is a maximal 50 point subset of the ASIA motor score for the representative arm and hand muscles.

Motor level is defined as the most caudal spinal level as indexed by the key muscle group for that level having a muscle strength of 3 or above while the key muscle for the spinal segment above is normal $(=5)$.

ASIA sensory score is calculated by testing a point on the dermatome for each spinal level from C2 to S4-5 for both light touch and pinprick sensation. Each point is assigned a score from 0 (absent sensation) through 1 (abnormal sensation) to 2 (normal sensation). This gives a possible maximum score of 56 on each side for a maximum total of 112 each for light touch and pinprick.

Sensory level is defined as the spinal segment corresponding with the most caudal dermatome having a normal score of $2 / 2$ for both pinprick and light touch.

Zone of partial preservation (ZPP) is only used when SCI is complete and refers to those segments below the neurological level of injury where there is some preservation of impaired motor or sensory function (usually, but not always, within a few segments of the neurological level).

The Benzel scale is a variant of the AIS classification that was used for the Sygen trial, in which the AIS grade $\mathrm{D}$ is expanded. Grade $1=\mathrm{AIS}$ A, Grade $2=\mathrm{AIS}$ B, Grade $3=$ AIS C, Grade 4= AIS D, Grade 5= AIS D, Grade $6=$ AIS D, Grade $7=$ AIS E. For a full description see Coleman and Geisler. ${ }^{21}$

\section{Acknowledgements}

We are grateful for the support of The International Campaign for Cures of spinal cord injury Paralysis (ICCP), which provided the funding for the authors' travel and accommodation expenses. The ICCP represents the following member organizations: Christopher Reeve Foundation (USA), Institut pour la Recherche sur la Moëlle Epinière (FRA), International Spinal Research Trust (UK), Japan Spinal Cord Foundation, Miami Project to Cure Paralysis (USA), Paralyzed Veterans of America (USA), Rick Hansen Man In Motion Foundation (CAN), SpinalCure Australia, and Spinal Research Fund of Australia. We thank the European Multicenter study in Spinal Cord Injury (EM-SCI) for sharing their data on spontaneous recovery after spinal cord injury. ICORD (International Collaboration On Repair Discoveries) in Vancouver provided all logistical coordination and support. All panel members (authors) volunteered their time and effort. Finally, we are most grateful for the input and constructive comments from a countless number of SCI investigators over the past 2.5 years.

\section{References}

1 Steeves J, Fawcett J, Tuszynski M. Report of International Clinical Trials Workshop on Spinal Cord Injury February 20-21, 2004, Vancouver, Canada. Spinal Cord 2004; 42: 591-597.

2 Bracken MB et al. A randomized, controlled trial of methylprednisolone or naloxone in the treatment of acute spinal cord injury. Results of the second National Acute Spinal Cord Injury Study. N Engl J Med 1990; 322: 1405-1411.

3 Bracken MB et al. Methylprednisolone or naloxone treatment after acute spinal cord injury: 1-year follow-up data. Results of the second national acute spinal cord injury study. J Neurosurg 1992; 76: 23-31.

4 Bracken MB, et al., for the National Acute Spinal Cord Injury Study. Administration of methylprednisolone for 24 or 48 hours or titilazad mesylate for 48 hours in the treatment of acute spinal cord injury. Results of the Third National Acute Spinal Cord Injury Randomized Controlled Trial. National Acute Spinal Cord Injury Study. JAMA 1997; 277: 1597-1604.

5 Bracken MB et al. Methylprednisolone or tirilazad mesylate administration after acute spinal cord injury: 1-year follow-up. Results of the third national acute spinal cord injury randomized controlled trial. J Neurosurg 1998; 89: 699-706.

6 Geisler FH, Coleman WP, Grieco G, Poonian D, the Sygen ${ }^{\mathbb{R}}$ Study Group. Measurements and recovery patterns in a multicenter study of acute spinal cord injury. Spine 2001; 26: S68-S86. 
7 Geisler FH, Coleman WP, Grieco G, Poonian D, the Sygen $\left.{ }^{(}\right)$Study Group. The Sygen ${ }^{\circledR}$ multicenter acute spinal cord injury study. Spine 2001; 26: S87-S98.

8 Tadié $\mathrm{M}$ et al. Early care and treatment with a neuroprotective drug, gacyclidine, in patients with acute spinal cord injury. Rachis 2003; 15: 363-376.

9 Steeves JD et al. Guidelines for the conduct of clinical trials for spinal cord injury (SCI) as developed by the ICCP Panel: Clinical trial outcome measures. Spinal Cord [E-pub ahead of print: 19 December 2006; doi:10.1038/ sj.sc.3102008].

10 Waters RL, Adkins RH, Yakura JS, Sie I. Motor and sensory recovery following complete tetraplegia. Arch Phys Med Rehabil 1993; 74: 242-247.

11 Marino RJ, Ditunno JF, Donovan WH, Maynard F. Neurologic recovery after traumatic spinal cord injury: Data from the Model Spinal Cord Injury Systems. Arch Phys Med Rehabil 1999; 80: 1391-1396.

12 Kirshblum S, Millis S, McKinley W, Tulsky D. Late neurologic recovery after traumatic spinal cord injury. Arch Phy Med Rehabil 2004; 85: 1811-1817.

13 Burns AS, Ditunno JF. Establishing prognosis and maximizing functional outcomes after spinal cord injury: a review of current and future directions in rehabilitation management. Spine 2001; 26: S137-S145.

14 Waters RL. Donald Munro lecture: functional and neurologic recovery following acute SCI. J Spinal Cord Med 1998; 21: 195-199.
15 Ditunno JF, Cohen ME, Hauck WW, Jackson AB, Sipski ML. Recovery of upper-extremity strength in complete and incomplete tetraplegia: a multicenter study. Arch Phys Med Rehabil 2000; 81: 389-393.

16 Fisher CG, Noonan VK, Smith DE, Wing PC, Dvorak MF, Kwon B. Motor recovery, functional status, and health-related quality of life in patients with complete spinal cord injuries. Spine 2005; 30: 2200-2207.

17 Ditunno JF, Stover SL, Freed MM, Ahn JH. Motor recovery of the upper extremities in traumatic quadriplegia: a multicenter study. Arch Phys Med Rehabil 1992; 73: 431-436.

18 Oleson CV, Burns AS, Ditunno JF, Geisler FH, Coleman WP. Prognostic value of pinprick preservation in motor complete, sensory incomplete spinal cord injury. Arch Phys Med Rehabil 2005; 86: 988-992.

19 Brown PJ, Marino RJ, Herbison, Ditunno Jr JF. The 72-h examination as a predictor of recovery in motor complete quadriplegia. Arch Phys Med Rehabil 1991; 72: 546-548.

20 Burns AS, Lee BS, Ditunno JF, Tessler A. Patient selection for clinical trials: the reliability of the early spinal cord injury examination. I Neurotrauma 2003; 20: 477-482.

21 Coleman WP, Geisler FH. Injury severity as primary predictor of outcome in acute spinal cord injury: retrospective results from a large multicenter clinical trial. Spine J 2004; 4: 373-378.

Supplementary Information accompanies the paper on the Spinal Cord website (http://www.nature.com/sc) 